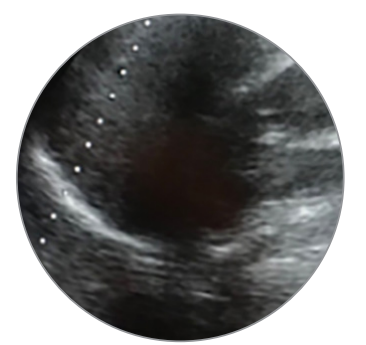

$\sum$

Palabras clave (DeCS)

Diafragma

Ultrasonido

Análisis estadístico

Correlación de datos

\section{Key words (MeSH)}

\section{Diaphragm}

Ultrasonics

Statistical analysis

Correlation of data
Médico especialista en Radiología e Imágenes Diagnósticas, Fundación Clínica Shaio. Bogotá, Colombia.

${ }^{2}$ Residente de Radiología, Universidad de La Sabana. Magister en Epidemiología, especialista en docencia universitaria. Bogotá, Colombia.

3Médico especialista en Radiología e Imágenes Diagnósticas, Fundación Clínica Shaio. Coordinador del área de Doppler de la Fundación Clínica Shaio. Bogotá, Colombia.

\title{
Excursión diafragmática en adultos sanos: valores de normalidad. Estudio de prevalencia analítica
}

\author{
Diaphragmatic Excursion in Healthy Adults: Normal Values. Analytical \\ Prevalence Study
}

Alfredo Zedan'

William Andrés Prada Mancilla ${ }^{2}$

Pedro Rey ${ }^{3}$

\section{Resumen}

Introducción: la excursión diafragmática por ultrasonido es una herramienta que permite valorar adecuadamente al paciente con patología crítica de la vía aérea, con derrame pleural y lesiones neurovasculares que generan patrones restrictivos sobre el tórax. Realizar la valoración dinámica del diafragma antes y después de un procedimiento permite conocer los cambios en la historia natural de la enfermedad. Por esto, es primordial conocer los valores de normalidad de la población de estudio, y cómo interfiere con las demás variables antropométricas de los pacientes. Materiales y métodos: estudio de prevalencia analítico para la medición de variables normales de la excursión diafragmática y la correlación con variables antropométricas de pacientes sin patología pulmonar o diafragmática. Resultados: muestra aleatoria simple de 50 diafragmas con una distribución del 68 \% de mujeres, con edad media de 39 años y una mediana del perímetro abdominal de 78 cm. El grosor del diafragma fue de 3,4 mm en inspiración y 2,6 mm en espiración. La media de la excursión diafragmática fue de $15 \mathrm{~mm}$. La correlación de Kendall entre la excursión y las variables antropométricas tuvo un valor de rho= 0,94 (Spearman). Conclusiones: Ios valores de normalidad de la excursión diafragmática son muy variables según la población estudiada, con relación clínica entre las variables antropométricas. Esto nos permite recomendar practicar una valoración dinámica del diafragma antes y después de cada procedimiento para evaluar los cambios significativos en los valores de excursión diafragmática.

\section{Summary}

Introduction: diaphragmatic excursion by ultrasound is a tool that allows to properly assess the patient with critical pathology of the airway, with pleural effusion and neurovascular injuries that generate restrictive patterns on the chest. Performing dynamic evaluation of the diaphragm before and after a procedure allows us to know the changes in the natural history of the disease. For this reason, it is essential to know the normal values of the study population, and how it interferes with the other anthropometric variables of the patients. Materials and methods: analytical prevalence study for the measurement of normal excursion variables and the correlation with anthropometric variables of patients without pulmonary or diaphragmatic pathology. Results: simple random sample of 50 diaphragms with a distribution of $68 \%$ of women, with a mean age of 39 years and a median of the abdominal perimeter of $78 \mathrm{~cm}$. The thickness of the diaphragm was $3.4 \mathrm{~mm}$ on inspiration and $2.6 \mathrm{~mm}$ on expiration, with a mean diaphragmatic excursion of $15 \mathrm{~mm}$. The Kendall correlation between the excursion and the anthropometric variables had a value of rho $=0.94$. Conclusions: the normal values of the diaphragmatic excursion are highly variable according to the population studied, with a clinical relationship between the anthropometric variables. This allows us to recommend that we should always perform a dynamic assessment of the diaphragm before and after each procedure in order to evaluate significant changes in diaphragmatic excursion values.

\section{Introducción}

La excursión diafragmática medida con ultrasonido ofrece varios beneficios para la evaluación de la función diafragmática y de su relación con la función pulmonar (figura 1). Existen publicaciones acerca del rendimiento diagnóstico del ultrasonido y de los valores críticos patológicos en diferentes grupos poblacionales; sin embargo, no se encuentran publicados valores nacionales normales que permitan direccionar el estrecho intervalo en el que se encuentran los valores patológicos en el diafragma, ya que se trata de valores en milímetros. Así mismo, no se conoce cómo varían estos valores en la población colombiana según la correlación con variables de los pacientes que puedan estar influenciando estos parámetros de normalidad. Por lo anterior, esta investigación pretende demostrar los valores normales de excursión diafragmática en el estudio por ultrasonido y su correlación con variables de interés, como las medidas antropométricas, en una población sin enfermedad pulmonar. 


\section{Materiales y métodos}

Se realizaron las ecografías con un equipo de ultrasonido Toshiba Aplio XG, en individuos sanos (sin patología descrita del diafragma) que se encontraban laborando, estudiando o eran pacientes para estudio de ultrasonido de abdomen en la institución en el momento de la investigación. La técnica para la realización del estudio fue: a los pacientes en decúbito supino se les examinó con dos transductores, convex y lineal de alta frecuencia, con maniobras dinámicas de inspiración y espiración en ambos diafragmas. Se utilizaron los modos B (brightness) y $\mathrm{M}$ (motion) para las medidas cuantitativas.

El reclutamiento se hizo así: Los individuos fueron invitados a participar en el estudio mediante invitación electrónica y el voz a voz por parte de los investigadores en el Departamento de Radiología, donde se llevaron a cabo posteriormente los estudio ultrasonográficos en áreas administrativas.

Para el cálculo de la muestra se realizó un análisis de muestreo probabilístico para análisis de medias para una población total de 2000 participantes que es el número de personas aproximado de la clínica. Se determinó un nivel de significancia del $95 \%$, una desviación estándar esperada de 0,04 y una precisión del 0,01 . Con esto se obtuvo un cálculo de 40 individuos y al adicionar un $10 \%$ de pérdida esperada estándar, se consideraron 50 diafragmas para el análisis.

Para el plan de análisis del estudio se planteó inicialmente un análisis univariado, el cual se estableció mediante tablas de frecuencia y porcentajes para las variables cualitativas, como el género. Por otro lado, para las variables cuantitativas, como la edad, la magnitud de la excursión y el grosor del diafragma, se establecieron medidas de tendencia central. Cada variable fue calculada independientemente, como la fracción de engrosamiento diafragmático. Si bien su cálculo es derivado de la relación en espiración e inspiración, los valores reportados son los de las medidas de tendencia central de los pacientes y no los del cálculo de sus variables relacionadas. Las variables cuantitativas se sometieron a pruebas de normalidad mediante el test de Shapiro-Wilk y Kolmogorov-Smirnov; Si presentaban distribución normal se utilizarían las medidas de tendencia central, desviación estándar y media, pero si la distribución era no normal, se presentarían la mediana y los valores mínimos y máximos. Para el desarrollo del objetivo específico de correlación entre la magnitud de la excursión diafragmática y las medidas antropométricas se realizó el test de correlación de Spearman.

Los investigadores recolectaron los datos en la fuente primaria, previo consentimiento informado y asentimiento de los pacientes. Posteriormente, para su análisis e interpretación se incluyeron en una base de datos en línea tipo format de Google, la cual fue exportada a los programas SPSS y STATA 13 para el análisis estadístico.

\section{Resultados}

En los pacientes evaluados se encontró una distribución homogénea de género con una proporción del $68 \%$ de mujeres, con una edad media de 39 (19-65) años.

El promedio del peso de los pacientes fue de $69 \mathrm{~kg}$, con una media de estatura de $165 \mathrm{~cm}$ aproximadamente. El perímetro abdominal osciló entre 62 y $97 \mathrm{~cm}$, con una mediana de $78 \mathrm{~cm}$ (tablas 1 y 2).

Tabla 1. Distribución de género

\begin{tabular}{|c|c|c|c|c|}
\hline Género & Frecuencia & Porcentaje & Porcentaje válido & Porcentaje acumulado \\
\hline Mujer & 34 & 68,0 & 68,0 & 68,0 \\
\hline Hombre & 16 & 32,0 & 32,0 & 100,0 \\
\hline Total & 50 & 100,0 & 100,0 & \\
\hline
\end{tabular}

Tabla 2. Medidas de tendencia central de variables cuantitativas

\begin{tabular}{|c|c|c|c|c|c|}
\hline & Media & Mediana & Máximo & Mínimo & $\begin{array}{l}\text { Desviación } \\
\text { estándar }\end{array}$ \\
\hline Edad & 39,52 & 38,50 & 65,00 & 19,00 & 14,67 \\
\hline Peso (kg) & 69,42 & 71,00 & 91,00 & 45,00 & 14,72 \\
\hline Talla $(\mathrm{cm})$ & 164,88 & 164,00 & 181,00 & 151,00 & 10,71 \\
\hline Perímetro abdominal (cm) & 79,46 & 78,00 & 97,00 & 62,00 & 10,60 \\
\hline $\begin{array}{l}\text { Grosor del diafragma en modo } B \text { en } \\
\text { espiración }(\mathrm{mm})\end{array}$ & 3,40 & 3,70 & 5,50 & 1,70 & 1,16 \\
\hline $\begin{array}{l}\text { Grosor del diafragma en modo } B \text { en } \\
\text { inspiración }(\mathrm{mm})\end{array}$ & 2,58 & 2,50 & 4,10 & 1,20 & 0,88 \\
\hline $\begin{array}{l}\text { Fracción del engrosamiento diafragmático } \\
(\%)\end{array}$ & 43,42 & 38,50 & 80,00 & 13,00 & 19,37 \\
\hline Excursión diafragmática media modo $\mathrm{M}$ & 14,58 & 15,00 & 19,00 & 10,00 & 2,14 \\
\hline Excursión diafragmática máxima modo M & 21,14 & 16,00 & 213,00 & 11,00 & 28,07 \\
\hline Velocidad de espiración en modo M & 6,19 & 1,90 & 218,00 & 0,80 & 30,57 \\
\hline
\end{tabular}




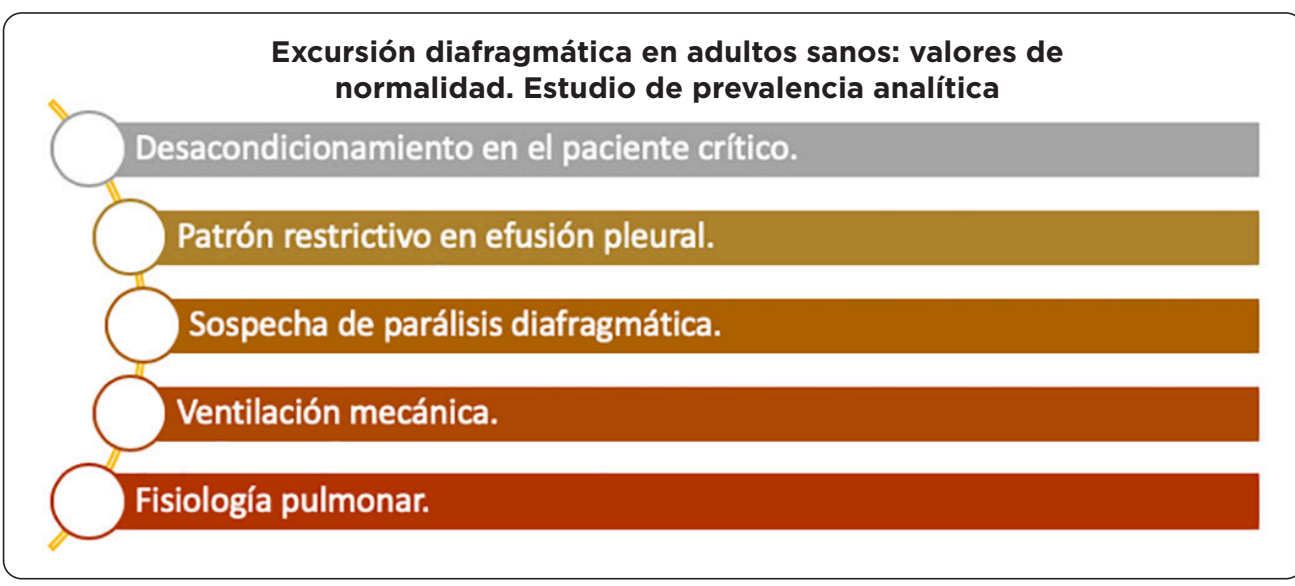

Figura 1. Esquema de utilidad de la valoración ecográfica en la excursión diafragmática

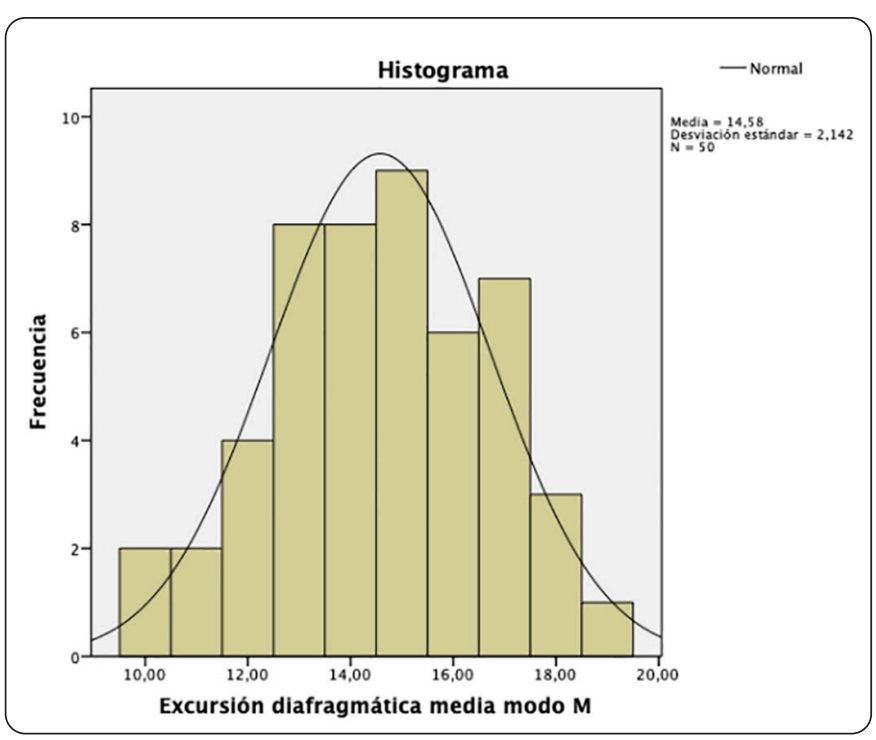

Figura 2. Distribución normal de la excursión diafragmática medida en modo $M$.

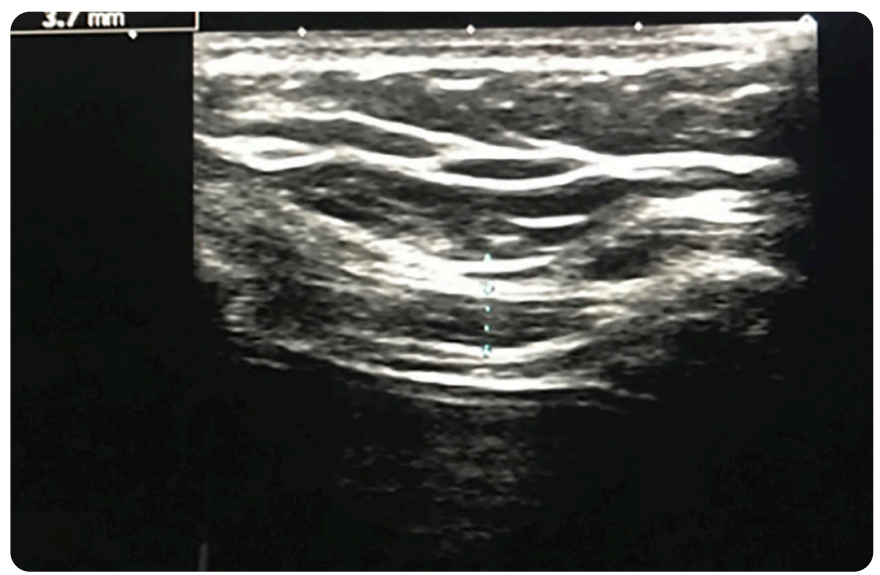

Figura 3. Grosor diafragmático medido en modo B

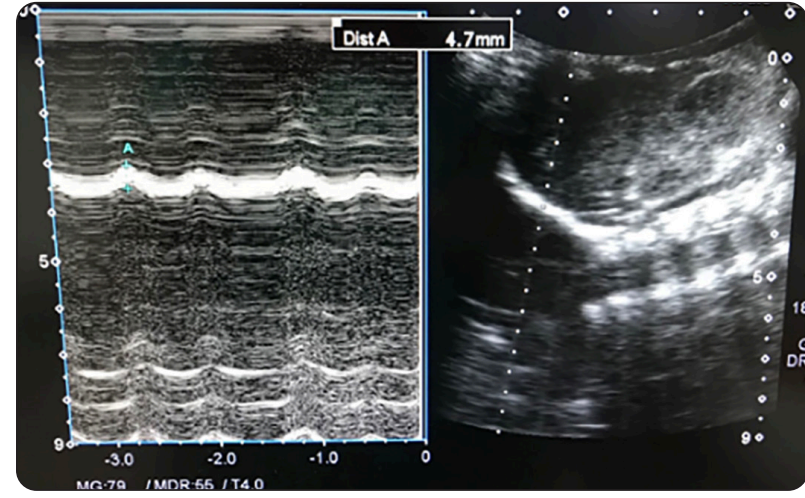

Figura 4. Grosor diafragmático medido en modo $\mathrm{M}$

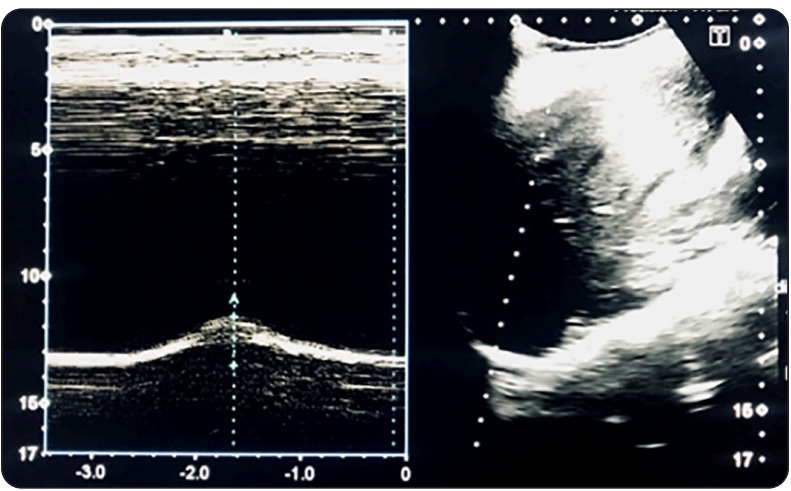

Figura 5. Excursión diafragmática derecha medida en modo M.

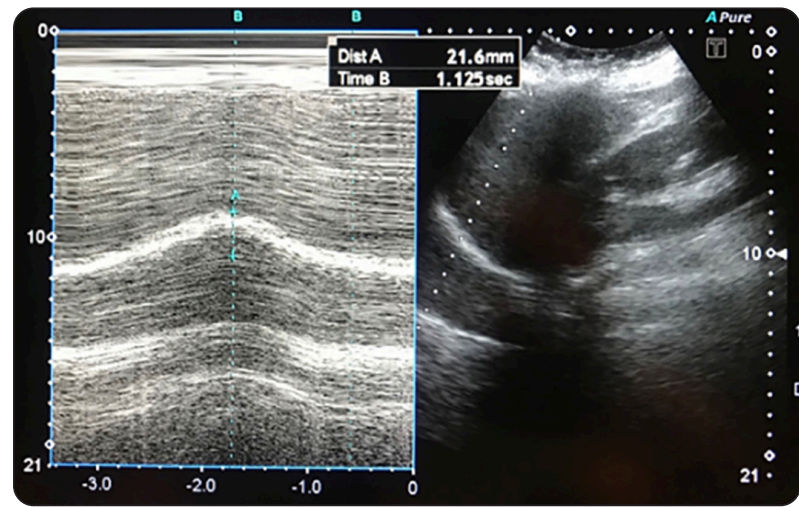

Figura 6. Excursión diafragmática izquierda medida en modo M. 


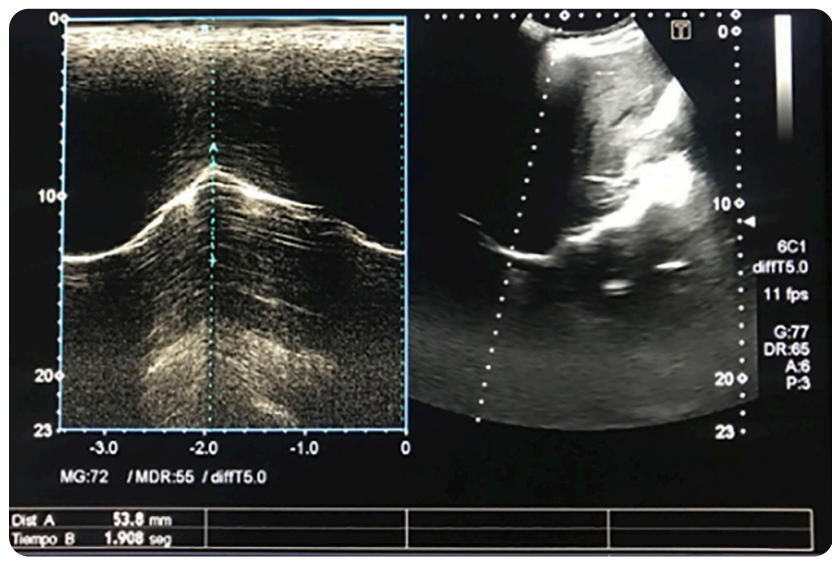

Figura 7. Tiempo de espiración de la excursión diafragmática.

\subsection{Resultados específicos de excursión diafragmática por ultrasonido}

La media del grosor del diafragma medido durante la espiración fue de 3,4 mm, mientras que en inspiración fue de 2,58 $\mathrm{mm}$; con una fracción de engrosamiento de $43 \%$ aproximadamente. La excursión diafragmática media medida en modo $\mathrm{M}$ fue de $15 \mathrm{~mm}$ y máxima de $21 \mathrm{~mm}$, y fue la única variable con distribución normal bajo la campana de Gauss (figura 2). La velocidad de espiración tuvo una mediana de 1,9 segundos (tabla 2 ).

\subsection{Análisis bivariado de variables independientes y excursión diafragmática}

El análisis avanzado de la relación entre las variables antropométricas de los pacientes y la excursión diafragmática mostró un valor de rho $=0.94$ (Spearman), con una relación clínica que evidenció entre mayor perímetro abdominal y mayor grosor del diafragma, menor excursión diafragmática. No se encontró relación significativa entre el perímetro abdominal y la velocidad de espiración ni la fracción del engrosamiento.

\section{Discusión}

La excursión diafragmática medida con ultrasonido tiene cada vez más utilidad en la valoración toracoabdominal de los pacientes. Tal vez la más utilizada sea en el paciente críticamente enfermo, para definir el desacondicionamiento del músculo, el cual es crucial para predecir el éxito de la extubación en los pacientes ventilados que cumplen criterios clínicos. La evidencia determina que los pacientes con criterios clínicos de extubación, pero con valores de desacondicionamiento diafragmático por ultrasonido, tienen mayor riesgo de falla ventilatoria temprana posterior a la extubación (1). A pesar de esto, se debe tener cuidado con las medidas estáticas de referencia en la literatura, porque para un paciente adulto mayor de baja talla y bajo peso, los valores anormales del paciente americano promedio son valores de normalidad, mientras que, si se tiene un valor de normalidad de referencia de este paciente antes de inicio de la terapia con ventilación mecánica, es más fácil establecer sus valores de corte para predecir el éxito de la extubación. En la valoración del desacondicionamiento físico del diafragma se ha establecido que una vez el paciente es intubado en la unidad de cuidado crítico, el grosor del diafragma disminuye de $6-7,5 \%$ por día $(2,3)$ y $3,4 \%$ en los niños (4). A pesar de esto, no se establece el valor inicial, sino los valores de seguimiento del estudio en estos pacientes. También se ha documentado que una fracción del engrosamiento menor al $20 \%$ o una excursión diafragmática inferior a $10 \mathrm{~mm}$ se establece como una disfunción diafragmática (5). Cuando la excursión diafragmática se encuentra entre 11 y $14 \mathrm{~mm}$ y la fracción de engrosamiento entre 30 y $36 \%$, se podría predecir una extubación exitosa con bajo riesgo de reintubación en las primeras horas (6-9). La investigación aquí descrita se ha realizado con pacientes sin patología pulmonar o diafragmática conocida, con resultados diferentes a lo publicado. El grosor del diafragma se puede medir en modo B, idealmente con transductor lineal; o en modo M, con un transductor de baja frecuencia. Se ha encontrado que los pacientes con valores inferiores a $2 \mathrm{~mm}$, tienen mayor tiempo de extubación (10). En este estudio se realizó la medición en modos $\mathrm{B}$ (figura 3), y M (figura 4), pero es más práctica la medición en modo $\mathrm{B}$, como lo describe la literatura (11). Se encontraron medias de grosor entre 2,6 y 3,4 mm, pero valores mínimos de 1,2 a 1,7, sobre todo en pacientes con menor perímetro abdominal; sin embargo, sin asociación estadísticamente significativa.

Por otro lado, el ultrasonido diafragmático también tiene utilidad en la valoración del derrame pleural, para definir cuál hemidiafragma está más comprometido cuando el derrame es bilateral. Es determinante definir qué hemitórax drenar cuando el derrame es bilateral, ya que no se pueden drenar los dos al mismo tiempo, por el riesgo de edema pulmonar por reexpansión. Esta problemática es muy frecuente en el paciente oncológico con derrame paraneumónico que requiere drenajes intermitentes con frecuencia. El ultrasonido diafragmático permite conocer cuál hemitórax tiene mayor restricción y cuál tiene su función pulmonar más afectada, y este último es el elegido para el drenaje percutáneo $(1,2)$.

Finalmente, la valoración de la hipotonía del diafragma en la sospecha de parálisis, es una utilidad importante del ultrasonido diafragmático, ya que, a diferencia de la fluoroscopia, permite evaluar en tiempo real la movilidad del diafragma. Adicionalmente, facilita la cuantificación los valores del movimiento diafragmático, compararlos con el lado contralateral, y hacer una valoración comparativa antes y después de que los pacientes son sometidos a plicatura quirúrgica.

La valoración de la excursión diafragmática en esta investigación tuvo una distribución normal (figura 2), con comportamiento de buena distribución que se acercó al parámetro poblacional. Se encontró que fue más práctica la valoración del hemidiafragma derecho (figura 5) que del izquierdo (figura 6), debido a que la ventana de insonación izquierda es más superior y posterior. Todos los valores de la muestra fueron mayores de $10 \mathrm{~mm}$, como lo describe la literatura; sin embargo, también se encontró una relación inversa entre el perímetro abdominal y la excursión, y es más difícil el movimiento diafragmático en pacientes con mayor perímetro. A pesar de esto, los pacientes con mayor perímetro, pero con mayor estatura, tienen mayor excursión diafragmática.

La fracción de engrosamiento diafragmático en esta investigación también tuvo un rango más amplio en relación con los valores en espiración e inspiración (tabla 2), y estuvo también en relación con el perímetro abdominal y estatura de los pacientes. Así mismo, en el tiempo de espiración hubo una varianza amplia, debido a que está 
en estrecha relación con el pico de excursión diafragmática (figura 7); sin embargo, el tiempo de espiración sirve para la valoración del intercambio gaseoso e indirectamente para evaluar el patrón restrictivo extrapulmonar de los pacientes, como el aumento de la presión intraabdominal. Debido a todo lo anterior, es muy importante conocer los valores antropométricos de los pacientes y tener siempre un valor inicial de medición para interpretar los valores patológicos en la predicción del desacondicionamiento del diafragma, ya que un valor de $1,7 \mathrm{~mm}$ en el grosor y una excursión diafragmática de $10 \mathrm{~mm}$ pueden ser normales en un paciente de baja estatura y con un perímetro abdominal menor, mientras que en un paciente de mayor estatura con perímetro abdominal amplio, es francamente patológico.

Limitaciones del estudio: Este estudio tiene como limitaciones en su diseño metodológico que no permite asociaciones causales debido a que es un corte transversal de la situación de pacientes sanos con medición de excursión diafragmática. Así mismo, tiene limitaciones del muestreo. Debido a lo anterior se recomienda el desarrollo de estudios con mayor muestra de análisis y de metodología analítica para la validación externa de los resultados y análisis planteados.

\section{Conclusión}

Los valores de normalidad en el grosor y la excursión diafragmática tienen un rango de normalidad amplio y se encuentran en relación con los valores antropométricos de los pacientes, por lo cual los investigadores recomiendan siempre tener un valor basal del grosor medido en modo $\mathrm{B}$, excursión diafragmática medida mediante en $\mathrm{M}$, fracción de engrosamiento y tiempo de espiración, para poder predecir los valores anormales en todos los estudios que se realicen a los pacientes durante la valoración dinámica en la evolución de la historia natural de la enfermedad.

\section{Referencias}

1. Turton P, ALAidarous S, Welters I. A narrative review of diaphragm ultrasound to predict weaning from mechanical ventilation: where are we and where are we heading? Ultrasound J. 2019;11(2).

2. Grosu HB, Lee YI, Lee J, Eden E, Eikermann M, Rose KM. Diaphragm muscle thinning in patients who are mechanically ventilated. Chest. 2012;142:1455-60.

3. Zambon M, Beccaria P, Matsuno J. Mechanical ventilation and diaphragmatic atrophy in critically ill patients: an ultrasound study. Crit Care Med. 2016;43:29-38.

4. Glau CL, Conlon TW, Himebauch AS, Yehya N, Weiss SL, Berg RA, et al. Progressive diaphragm atrophy in pediatric acute respiratory failure. Pediatr Crit Care Med. 2018;19:406-11.

5. Gursel G, Inci K, Alasgarova Z. Can diaphragm dysfunction be reliably evaluated with pocket-sized ultrasound devices in intensive care Unit? Crit Care Res Pract. 2018; article ID: 5192647

6. Kim Y, Hee HJ, Suh J, Hong RSB, Koh Y, Lim CM. Diaphragm dysfunction assessed by ultrasonography: influence on weaning from mechanical ventilation. Crit Care Med. 2011;39:2627-30

7. Jiang JR, Tsai TH, Jerng JS, Yu CJ, Wu HD, Yang PC. Ultrasonographic evaluation of liver/spleen movements and extubation outcome. Chest. 2004;126:179-85.

8. Dinino E, Gartman EJ, Sethi JM, Mccool D. Diaphragm ultrasound as a predictor of successful extubation from mechanical ventilation. Thorax. 2014:69:423-7.

9. Ferrari G, De Filippi G, Fabrizio E, Panero F, Volpicelli G, Apra F. Diaphragm ultrasound as a new index of discontinuation from mechanical ventilation. Crit Ultrasound J. 2014;6:8

10. Sharma A, Karna ST, Tandon M, Pandey CK, Chaturvedi R, Vyas V, et al. Use of ultrasound-guided preoperative diaphragmatic thickness as a predictor of postoperative weaning failure in recipients and donors scheduled for living donor liver transplant surgery. Saudi J Anaesth. 2018;12:406-11.

11. Dres M, Goligher EC, Dubé B-P, Morawiec E, Dangers L, Reuter D, et al. Diaphragm function and weaning from mechanical ventilation: an ultrasound and phrenic nerve stimulation clinical study. Ann Intensive Care. 2018;8:53

\section{Correspondencia}

William Andrés Prada Mancilla

Av. calle 170 \# 56-45, apto 2004, Edificio Belgrano

Bogotá, Colombia

wpradamancilla@gmail.com

Recibido para evaluación: 18 de julio de 2020

Aceptado para publicación: 15 de septiembre de 2020 